PHYSICAL REVIEW D 96, 011101(R) (2017)

\title{
Observation of the $B^{+} \rightarrow D^{*-} K^{+} \pi^{+}$decay
}

\author{
R. Aaij et al. \\ (LHCb Collaboration)
}

(Received 27 April 2017; published 19 July 2017)

\begin{abstract}
The $B^{+} \rightarrow D^{*-} K^{+} \pi^{+}$decay potentially provides an excellent way to investigate charm meson spectroscopy. The decay is searched for in a sample of proton-proton collision data collected with the LHCb detector at center-of-mass energies of 7 and $8 \mathrm{TeV}$, corresponding to an integrated luminosity of $3 \mathrm{fb}^{-1}$. A clear signal is observed, and the ratio of its branching fraction to that of the $B^{+} \rightarrow D^{*-} \pi^{+} \pi^{+}$ normalization channel is measured to be $\mathcal{B}\left(B^{+} \rightarrow D^{*-} K^{+} \pi^{+}\right) / \mathcal{B}\left(B^{+} \rightarrow D^{*-} \pi^{+} \pi^{+}\right)=(6.39 \pm 0.27 \pm 0.48) \times$ $10^{-2}$, where the first uncertainty is statistical and the second is systematic. This is the first observation of the $B^{+} \rightarrow D^{*-} K^{+} \pi^{+}$decay.
\end{abstract}

DOI: 10.1103/PhysRevD.96.011101

The $B \rightarrow D^{(*)} h h^{\prime}$ decays, where $h^{(\prime)}=\pi, K$, provide an excellent way to investigate the spectroscopy of excited charm mesons. The constrained initial and final states lead to comparatively low backgrounds and excellent mass resolution, and furthermore amplitude analysis can be used to determine the quantum numbers of any intermediate resonant states through their angular distributions. By contrast, very large yields are available through inclusive production of excited charm states, but studies of such processes cannot in general result in unambiguous determinations of quantum numbers, and the sizable backgrounds tend to lead to large systematic uncertainties.

The amplitude analysis approach has been pursued extensively for $B \rightarrow D h h^{\prime}$ decays. For the cases where both $h h^{\prime}$ particles are pions, the $B^{+} \rightarrow D^{-} \pi^{+} \pi^{+}$and $B^{0} \rightarrow$ $\bar{D}^{0} \pi^{+} \pi^{-}$decays have been studied by the Belle $[1,2]$, $B A B A R[3,4]$ and $\mathrm{LHCb}[5,6]$ collaborations. Regarding modes with a kaon in the final state, detailed analyses of $B^{+} \rightarrow D^{-} K^{+} \pi^{+}[7], B^{0} \rightarrow \bar{D}^{0} K^{+} \pi^{-}[8]$ and $B_{s}^{0} \rightarrow \bar{D}^{0} K^{-} \pi^{+}$ $[9,10]$ decays have been performed by $\mathrm{LHCb}$. In spite of the Cabibbo suppression of the $B^{+}$and $B^{0}$ decays to the final states containing kaons compared to those with only pions, sufficiently large samples can be obtained to provide useful independent measurements of the properties of excited charm mesons.

The above-mentioned decays are, however, only sensitive to resonant states with natural spin-parity, i.e. with $J^{P}$ in the series $0^{+}, 1^{-}, 2^{+}, 3^{-}, \ldots$, as only those states can decay strongly to two pseudoscalar mesons. Relatively little information exists on the states with unnatural spinparity. Apart from work on the $B^{+} \rightarrow D^{*-} \pi^{+} \pi^{+}$mode by Belle [1], there has been no experimental study of the

${ }^{*}$ Full author list given at end of the article.

Published by the American Physical Society under the terms of the Creative Commons Attribution 4.0 International license. Further distribution of this work must maintain attribution to the author(s) and the published article's title, journal citation, and DOI. resonant substructure of $B \rightarrow D^{*} h h^{\prime}$ decays. Studies of inclusive production of $D^{*} \pi$ resonances in $e^{+} e^{-}$and $p p$ collisions have been made by BABAR [11] and $\mathrm{LHCb}$ [12], respectively, but more detailed investigations are necessary to understand the spectrum of states.

Decays of the form $B \rightarrow D^{(*)} K \pi$ are also important in the context of determining the angle $\gamma$ of the CabibboKobayashi-Maskawa (CKM) quark mixing matrix [13,14]. Sensitivity to $\gamma$ arises when amplitudes proportional to the CKM matrix elements $V_{u b} V_{c s}^{*}$ and $V_{c b} V_{u s}^{*}$ interfere, and so the $D^{(*)}$ meson must decay into a final state accessible to both charm flavor eigenstates. This is not possible for $D^{(*)+}$ decays. However, the relative rates of $B^{+} \rightarrow D^{(*)+} K^{+} \pi^{-}$ and $B^{+} \rightarrow D^{(*)-} K^{+} \pi^{+}$decays through an intermediate $D^{(*) \pm} \pi^{\mp}$ resonance can be used to determine the relative magnitude of the two amplitudes [15], as was recently done for $B^{+} \rightarrow D^{ \pm} K^{+} \pi^{\mp}$ decays [7,16]. This information may subsequently be used as an external constraint in a determination of $\gamma$ from decays of the same resonance in the $B^{+} \rightarrow D^{(*)} K^{+} \pi^{0}$ final state. Moreover, as an increasingly wide range of decays are being used to obtain constraints on $\gamma$ [17], it is important to improve knowledge of modes such as $B \rightarrow D^{*} h h^{\prime}$ which may cause backgrounds and hence systematic uncertainties in the analyses.

In this paper, the first search for the $B^{+} \rightarrow D^{*-} K^{+} \pi^{+}$ decay is presented. The $D^{*-}$ meson is reconstructed through its decay to $\bar{D}^{0} \pi^{-}$with $\bar{D}^{0} \rightarrow K^{+} \pi^{-}$. The topologically similar $B^{+} \rightarrow D^{*-} \pi^{+} \pi^{+}$decay is used as a control channel and for normalization of the branching fraction measurement. The leading diagram for $B^{+} \rightarrow D^{*-} K^{+} \pi^{+}$ and $D^{*-} \pi^{+} \pi^{+}$decays is shown in Fig. 1. The inclusion of charge-conjugate processes is implied throughout the paper. The analysis is based on procedures used for previous analyses of similar decay modes [6,7]. An important feature is that signal decays have a narrow peak in the distribution of $\Delta m$, the difference between the $D^{*-}$ and $\bar{D}^{0}$ candidate masses; imposing a requirement on $\Delta m$ greatly reduces the range of possible sources of background. 


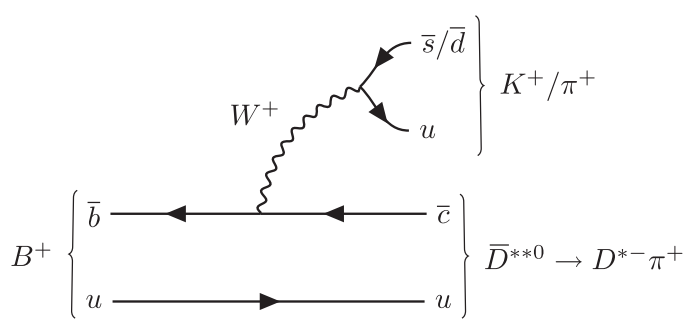

FIG. 1. Leading diagram for $B^{+} \rightarrow D^{*-} K^{+} \pi^{+}$and $D^{*-} \pi^{+} \pi^{+}$ decays, where the $D^{*-} \pi^{+}$system is produced through the decay of an excited charm state denoted $\bar{D}^{* * 0}$.

The analysis is based on a sample of proton-proton collision data collected with the LHCb detector at centerof-mass energies of 7 and $8 \mathrm{TeV}$, corresponding to an integrated luminosity of $3 \mathrm{fb}^{-1}$. The LHCb detector $[18,19]$ is a single-arm forward spectrometer covering the pseudorapidity range $2<\eta<5$, designed for the study of particles containing $b$ or $c$ quarks. The detector includes a highprecision tracking system consisting of a silicon-strip vertex detector surrounding the $p p$ interaction region, a large-area silicon-strip detector located upstream of a dipole magnet with a bending power of about $4 \mathrm{Tm}$ and three stations of silicon-strip detectors and straw drift tubes placed downstream of the magnet. The tracking system provides a measurement of momentum, $p$, of charged particles with relative uncertainty that varies from $0.5 \%$ at low momentum to $1.0 \%$ at $200 \mathrm{GeV} / c$. The minimum distance of a track to a primary vertex (PV), the impact parameter (IP), is measured with a resolution of $\left(15+29 / p_{\mathrm{T}}\right) \mu \mathrm{m}$, where $p_{\mathrm{T}}$ is the component of the momentum transverse to the beam, in $\mathrm{GeV} / c$. Different types of charged hadrons are distinguished using information from two ring-imaging Cherenkov detectors. Photons, electrons and hadrons are identified by a calorimeter system consisting of scintillating-pad and preshower detectors, an electromagnetic calorimeter and a hadronic calorimeter. Muons are identified by a system composed of alternating layers of iron and multiwire proportional chambers.

Online event selection is performed by a trigger, which consists of a hardware stage, based on information from the calorimeter and muon systems, followed by a software stage, in which all tracks with $p_{\mathrm{T}}>500(300) \mathrm{MeV} / c$ are reconstructed for data collected in 2011 (2012). At the hardware trigger stage, events are required to contain either a muon with high transverse momentum or a particle that deposits high transverse energy in the calorimeters. For hadrons, the transverse energy threshold is typically $3.5 \mathrm{GeV}$. The software trigger used in the analysis reported in this paper requires a two-, three- or four-track secondary vertex with significant displacement from any PV. At least one charged particle must have $p_{\mathrm{T}}$ above a threshold of $1.7(1.6) \mathrm{GeV} / c$ in the $\sqrt{s}=7$ (8) $\mathrm{TeV}$ data. This particle must also be inconsistent with originating from any $\mathrm{PV}$, as quantified through the difference in the vertex fit $\chi^{2}$ of a given $\mathrm{PV}$ reconstructed with and without the considered particle $\left(\chi_{\mathrm{IP}}^{2}\right)$. A multivariate algorithm [20] is used for the identification of secondary vertices consistent with the decay of a $b$ hadron. In the offline selection, the objects that fired the trigger are associated with reconstructed particles. Selection requirements can therefore be made not only on the particular trigger that fired, but on whether the decision was due to the signal candidate, other particles produced in the $p p$ collision, or a combination of both [21]. Candidates are retained from events in which the hardware trigger is caused either by the signal candidate or by other particles in the event. In the former case, it is further required that the trigger is caused by the deposits of the signal decay products in the calorimeters. It is also required that the software trigger decision must have been caused entirely by tracks that form the signal candidate.

Simulated events are used to characterize the detector response to signal and certain types of background events. In the simulation, $p p$ collisions are generated using PYTHIA [22] with a specific $\mathrm{LHCb}$ configuration [23]. Decays of hadronic particles are described by EVTGEN [24], in which final-state radiation is generated using Рнотоs [25]. The interaction of the generated particles with the detector and its response are implemented using the GEANT4 toolkit [26] as described in Ref. [27].

Candidates consistent with the decay chains $B^{+} \rightarrow$ $D^{*-} K^{+} \pi^{+}$and $B^{+} \rightarrow D^{*-} \pi^{+} \pi^{+}$, with $D^{*-} \rightarrow \bar{D}^{0} \pi^{-}$and $\bar{D}^{0} \rightarrow$ $K^{+} \pi^{-}$, are selected. The criteria for $B^{+} \rightarrow D^{*-} K^{+} \pi^{+}$and $B^{+} \rightarrow D^{*-} \pi^{+} \pi^{+}$candidates are identical, except for particle identification requirements (discussed below). Loose initial selection requirements on the quality of the tracks combined to form the $B^{+}$candidate, as well as on their $p, p_{\mathrm{T}}$ and $\chi_{\mathrm{IP}}^{2}$, are applied. The $\bar{D}^{0}$ candidate must have invariant mass within $\pm 100 \mathrm{MeV} / c^{2}$ of the known $\bar{D}^{0}$ mass [28]. Further requirements are imposed on the vertex quality $\left(\chi_{\mathrm{vtx}}^{2}\right)$ and flight distance of the $B^{+}$and $\bar{D}^{0}$ candidates from the PV with which they have the smallest $\chi_{\mathrm{IP}}^{2}$ (for the $B^{+}$ candidate, this is referred to as the associated PV). The $B^{+}$ candidate must also satisfy requirements on its invariant mass and on the cosine of the angle between the momentum vector and the line joining the $B$ vertex to the associated PV. The value of $\Delta m$ is required to be less than $5 \mathrm{MeV} / c^{2}$ from the known difference between the $D^{*-}$ and $\bar{D}^{0}$ masses [28].

A neural network [29] is used to further separate signal from background. The network is trained using a simulation sample to represent signal and data from a $D^{*-} K^{+} \pi^{+}$ mass sideband region to represent background. The network exploits differences between signal and background in the distributions of 16 input variables related to the kinematics and topology of the decay. The most discriminatory variables are the $B^{+}$candidate $\chi_{\mathrm{vtx}}^{2}$ and quantities related to the characteristic flight distances of the $B^{+}$and $\bar{D}^{0}$ mesons. It is verified that none of the input variables, nor the neural network output, are strongly correlated with the $B^{+}$candidate mass or with position in the phase space 
of the $B^{+}$meson decay. The selection requirement on the neural network output is optimized using a figure of merit that does not depend on the assumed signal branching fraction [30]. In this procedure, the relative efficiency of the neural network output requirement is determined from simulation, while the expected background under the signal peak in the $B^{+}$candidate mass is obtained by extrapolating from a $D^{*-} K^{+} \pi^{+}$mass sideband region. The same requirement is applied to both $D^{*-} K^{+} \pi^{+}$and $D^{*-} \pi^{+} \pi^{+}$candidates. The combined efficiency of the geometrical acceptance, online and offline selection (excluding particle identification) requirements is around $0.5 \%$ for both $D^{*-} K^{+} \pi^{+}$and $D^{*-} \pi^{+} \pi^{+}$final states.

Information from the ring-imaging Cherenkov detectors is combined with input from other subdetectors into variables designed to distinguish kaons from pions [31]. Requirements on the values of these variables for the pions and kaons originating directly from the $B^{+}$decay and for the kaon from the $\bar{D}^{0}$ decay are imposed. These are optimized using the same figure of merit as for the neural network output, with the signal efficiency determined from high-yield control samples of kaons and pions weighted to match the kinematic properties of signal decays. Application of the same procedure to the pions from the $D^{*-}$ and $\bar{D}^{0}$ decays indicates that no requirement is needed on the particle identification information associated with these particles. The combined efficiency of the particle identification requirements is about $60 \%$ for the $B^{+} \rightarrow$ $D^{*-} K^{+} \pi^{+}$decay and about $85 \%$ for the $B^{+} \rightarrow D^{*-} \pi^{+} \pi^{+}$ decay. The $\pi \rightarrow K$ misidentification rate is below $0.3 \%$.

Final-state particles from true $B^{0} \rightarrow D^{*-} \pi^{+}$decays can be combined with random pions to form a background which has a broad peak in the $B^{+}$candidate mass above the signal region in the normalization channel. In order to simplify the modeling of the background in this region, candidates with $D^{*-} \pi^{+}$invariant mass in the range $5200<$ $m\left(D^{*-} \pi^{+}\right)<5400 \mathrm{MeV} / c^{2}$ are vetoed. This requirement effectively removes the $B^{0} \rightarrow D^{*-} \pi^{+}$background with negligible loss of signal. No veto is applied to remove the similar background from $B^{0} \rightarrow D^{*-} K^{+}$decays as this is found to have negligible effect on the analysis. Following all selection requirements, fewer than $2 \%$ of events contain more than one candidate; all are retained. The associated systematic uncertainty is negligible.

Extended maximum likelihood fits to the distributions of candidates in $B^{+}$candidate mass are used to determine the yields of $B^{+} \rightarrow D^{*-} K^{+} \pi^{+}$and $B^{+} \rightarrow D^{*-} \pi^{+} \pi^{+}$ decays. The fits contain components to describe the signals, combinatorial background and partially reconstructed backgrounds. The latter are decays of the type $B \rightarrow D^{*-} h^{+} h^{\prime+} X$, where $X$ represents an additional particle that has not been included in the reconstructed decay chain. The fit to the $B^{+} \rightarrow D^{*-} K^{+} \pi^{+}$candidates also includes a component for cross-feed due to misidentified $B^{+} \rightarrow D^{*-} \pi^{+} \pi^{+}$decays.
The signal shapes are modeled by the sum of two Crystal Ball (CB) functions [32], which share a common peak position and have tails on opposite sides. The ratio of widths of the $\mathrm{CB}$ shapes and the fraction of entries in the narrower $\mathrm{CB}$ shape are constrained within their uncertainties to the values found in fits to simulated signal samples. The tail parameters of the $\mathrm{CB}$ shapes are fixed to those found in simulation. The combinatorial background in both samples is modeled with an exponential function. Partially reconstructed background is modeled by the convolution of a Gaussian with an ARGUS function [33], as this shape has been previously found to provide a good description of the kinematic limit for this component near $m_{B}-m_{\pi}$ [34,35]. The cross-feed background is modeled with a $\mathrm{CB}$ function with parameters obtained from a fit to $B^{+} \rightarrow D^{*-} \pi^{+} \pi^{+}$data reconstructed with the kaon mass assigned to one of the daughters, weighted according to the misidentification probability obtained from control samples.

The results of the fits are shown in Fig. 2. The fit to the $D^{*-} K^{+} \pi^{+}$sample has nine free parameters, which are the signal yield (744 \pm 29 ), the yields of the three background components, the peak position and width parameter of the signal shape, the slope of the combinatorial background and the two shape parameters of the partially reconstructed background. The fit to the $D^{*-} \pi^{+} \pi^{+}$sample has one fewer free parameter as no cross-feed component is included, and gives a signal yield of $17450 \pm 140$. The fit procedure is validated with ensembles of pseudoexperiments; any possible bias on the fitted yields is found to be negligible.

The ratio of branching fractions for $B^{+} \rightarrow D^{*-} K^{+} \pi^{+}$ and $B^{+} \rightarrow D^{*-} \pi^{+} \pi^{+}$decays is calculated by applying event-by-event efficiency corrections as a function of position in the $B^{+}$decay phase space,

$$
\frac{\mathcal{B}\left(B^{+} \rightarrow D^{*-} K^{+} \pi^{+}\right)}{\mathcal{B}\left(B^{+} \rightarrow D^{*-} \pi^{+} \pi^{+}\right)}=\frac{N^{\text {corr }}\left(B^{+} \rightarrow D^{*-} K^{+} \pi^{+}\right)}{N^{\text {corr }}\left(B^{+} \rightarrow D^{*-} \pi^{+} \pi^{+}\right)},
$$

where $N^{\text {corr }}=\sum_{i} W_{i} / \epsilon_{i}$ is the efficiency-corrected yield. Here the index $i$ runs over all candidates in the fitted data sample, $W_{i}$ is the signal weight for candidate $i$ and is determined using the sPlot procedure [36] from the fits in Fig. 2, and $\epsilon_{i}$ is the efficiency for candidate $i$. The efficiencies are evaluated including contributions from the LHCb detector acceptance, selection and trigger. The acceptance and most selection efficiencies are calculated from simulated samples with, where appropriate, datadriven corrections applied, while the particle identification efficiency is determined from control samples [31]. The phase space for a $P \rightarrow V P P$ decay, where $V(P)$ indicates a vector (pseudoscalar) particle, has four degrees of freedom, but for $B^{+} \rightarrow D^{*-} K^{+} \pi^{+}$it is found that the efficiency depends strongly only on the squares of the two-body invariant masses $m^{2}\left(D^{*-} \pi^{+}\right)$and $m^{2}\left(K^{+} \pi^{+}\right)$. Similarly for $B^{+} \rightarrow D^{*-} \pi^{+} \pi^{+}$decays, dependence of the efficiency on $m^{2}\left(D^{*-} \pi^{+}\right)_{\min }$ and $m^{2}\left(\pi^{+} \pi^{+}\right)$is accounted for, where 

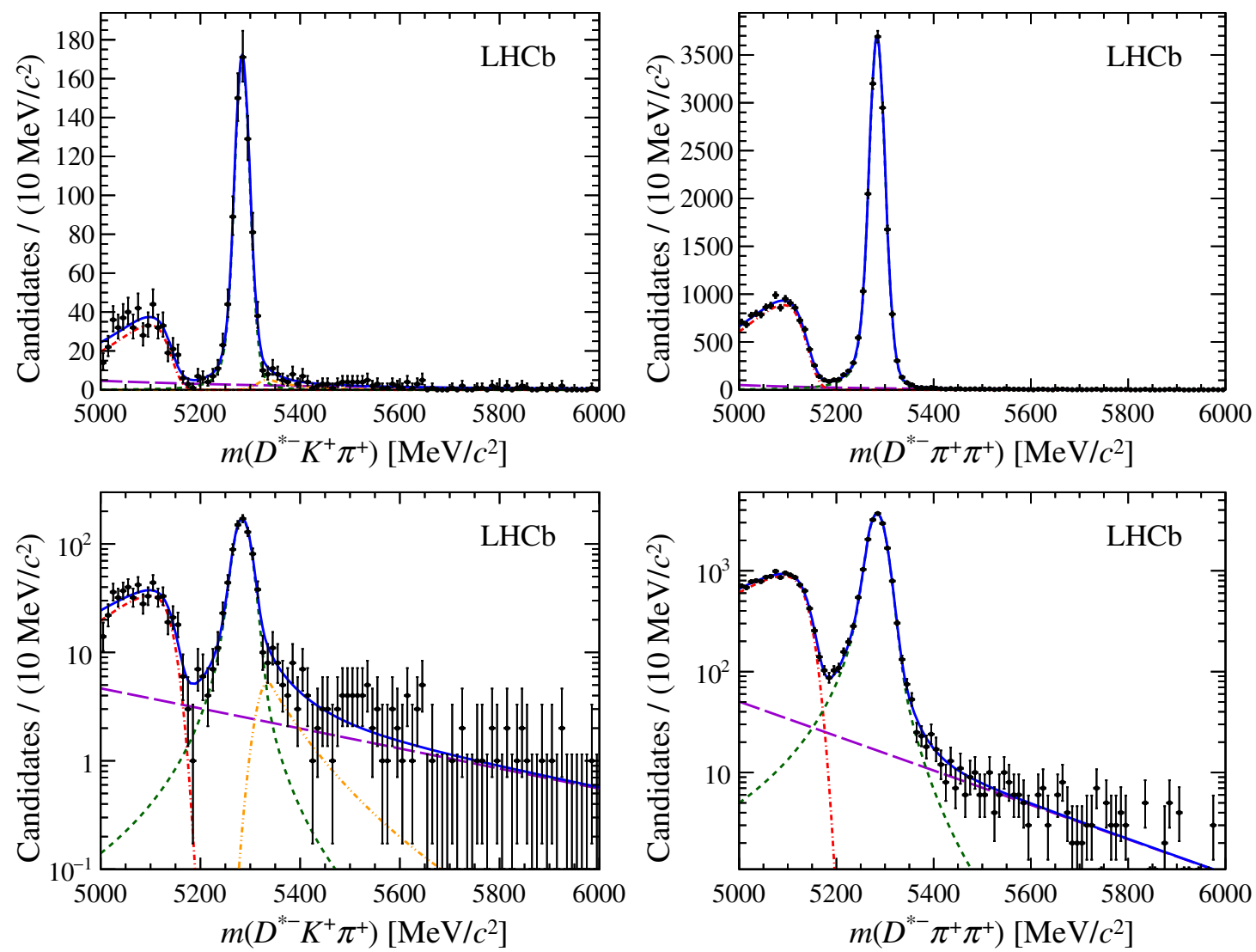

FIG. 2. Fits to $B$ candidate mass distributions for (left) $D^{*-} K^{+} \pi^{+}$and (right) $D^{*-} \pi^{+} \pi^{+}$samples with (top) linear and (bottom) logarithmic $y$-axis scales. The individual components are (solid blue) total fit function, (dashed green) signal shape, (long-dashed violet) combinatorial background, (dot dashed red) partially reconstructed background and (double-dot dashed orange) $D^{*-} \pi^{+} \pi^{+}$to $D^{*-} K^{+} \pi^{+}$ cross-feed.

$m^{2}\left(D^{*-} \pi^{+}\right)_{\min }$ indicates that the smaller of the two possible $m^{2}\left(D^{*-} \pi^{+}\right)$combinations is taken. The other two degrees of freedom in the phase space are related to the orientation of the $D^{*-} \rightarrow \bar{D}^{0} \pi^{-}$decay relative to the plane defined by the $B^{+} \rightarrow D^{*-} h^{+} h^{\prime+}$ decay. Possible variation of the efficiency with these variables is accounted for as a source of systematic uncertainty.

The background-subtraction and efficiency-correction procedures used to determine the values of $N^{\text {corr }}$ also allow the phase-space distributions of decays to be examined. The projection of the $D^{*-} K^{+} \pi^{+}$data onto $m\left(D^{*-} \pi^{+}\right)$is shown in Fig. 3. The asymmetric peak is indicative of the presence of contributions from both the $\bar{D}_{1}(2420)^{0}$ and $\bar{D}_{1}^{\prime}(2430)^{0}$ states [1]. A detailed investigation of the distribution of decays across the phase space is left for future study.

The statistical uncertainty evaluated from Eq. (1) includes contributions from the weighting and from the floated shape parameters in the fit [37]. Systematic uncertainties are assigned due to approximations made in the fit used to determine the yields and due to uncertainties in the efficiency. Variations of the fit model are made by modifying fixed parameters within their uncertainties, replacing the shapes used to describe each component with alternative functions, and, in the fit to the $D^{*-} \pi^{+} \pi^{+}$sample, introducing a component to account for cross-feed from $B^{+} \rightarrow$ $D^{*-} K^{+} \pi^{+}$decays. Uncertainties on the efficiency arise due to the limited size of the simulation samples, possible variation of the efficiency with $D^{*-}$ decay angles, possible imprecision of the data-driven method to determine particle

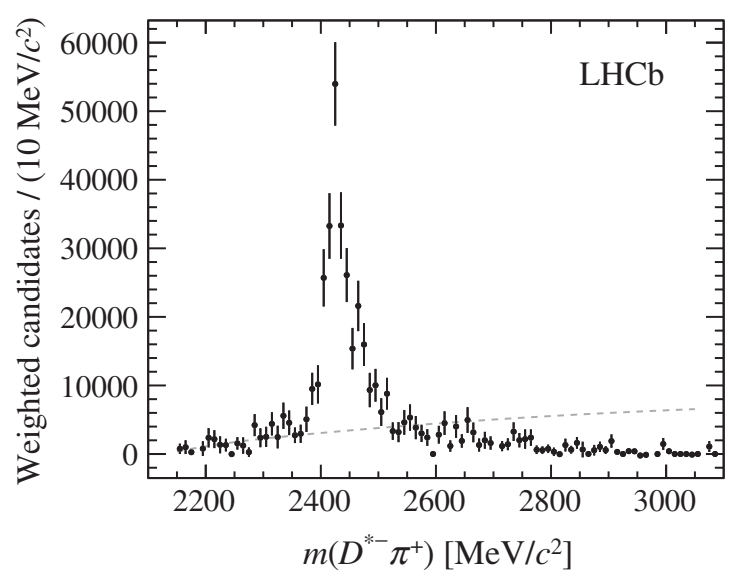

FIG. 3. Background-subtracted [36] and efficiency-corrected $m\left(D^{*-} \pi^{+}\right)$distribution from $B^{+} \rightarrow D^{*-} K^{+} \pi^{+}$decays. The grey dashed line illustrates a phase-space distribution, normalized to the same number of weighted candidates. 
TABLE I. Systematic uncertainties on the ratio of branching fractions.

\begin{tabular}{lc}
\hline \hline Source & Uncertainty \\
\hline Fit model & $3.3 \%$ \\
Simulation sample sizes & $5.4 \%$ \\
Efficiency variation with decay angles & $0.3 \%$ \\
Particle identification efficiency & $2.2 \%$ \\
Phase space vetoes & $3.2 \%$ \\
Total & $7.4 \%$ \\
\hline \hline
\end{tabular}

identification efficiencies and due to selection requirements that remove particular regions of phase space. The magnitudes of each of these contributions are summarized in Table I. The total systematic uncertainty on the ratio of branching fractions is $7.4 \%$.

The ratio of branching fractions is determined from Eq. (1) to be

$$
\frac{\mathcal{B}\left(B^{+} \rightarrow D^{*-} K^{+} \pi^{+}\right)}{\mathcal{B}\left(B^{+} \rightarrow D^{*-} \pi^{+} \pi^{+}\right)}=(6.39 \pm 0.27 \pm 0.48) \times 10^{-2},
$$

where the first uncertainty is statistical and the second is systematic. This constitutes the first observation of the $B^{+} \rightarrow D^{*-} K^{+} \pi^{+}$decay. The change in $\sqrt{-2 \ln \mathcal{L}}$ between fits with and without the signal component included, where $\mathcal{L}$ is the fit likelihood modified to account for systematic uncertainties that affect the yield, gives a value of 24 , showing clearly that the significance is far in excess of the $5 \sigma$ threshold normally used to claim observation.

In summary, the $B^{+} \rightarrow D^{*-} K^{+} \pi^{+}$decay has been observed for the first time in a data sample corresponding to $3 \mathrm{fb}^{-1}$ of integrated luminosity recorded with the $\mathrm{LHCb}$ detector. The ratio of the $B^{+} \rightarrow D^{*-} K^{+} \pi^{+}$and $B^{+} \rightarrow$ $D^{*-} \pi^{+} \pi^{+}$branching fractions has been measured, and has a value at the level naïvely expected due to the relative Cabibbo suppression of the former decay, $\left|V_{u s} / V_{u d}\right|^{2} \approx$ $5.3 \times 10^{-2}$. The measurements that comprise the current world average value $\mathcal{B}\left(B^{+} \rightarrow D^{*-} \pi^{+} \pi^{+}\right)=(1.35 \pm 0.22) \times$ $10^{-3}[1,28]$ all assume equal production of $B^{+} B^{-}$and $B^{0} \quad \bar{B}^{0}$ at the $\Upsilon(4 S)$ resonance. Using this value and correcting it with the latest result on $\Gamma\left(\Upsilon(4 S) \rightarrow B^{+} B^{-}\right) /$ $\Gamma\left(\Upsilon(4 S) \rightarrow B^{0} \bar{B}^{0}\right)[28]$ results in

$$
\mathcal{B}\left(B^{+} \rightarrow D^{*-} K^{+} \pi^{+}\right)=(8.2 \pm 0.3 \pm 0.6 \pm 1.3) \times 10^{-5},
$$

where the third uncertainty is due to the precision of the knowledge of the normalization channel branching fraction. Inspection of the phase-space distribution of signal decays confirms that this mode can be used to investigate charm meson spectroscopy.

\section{ACKNOWLEDGMENTS}

We express our gratitude to our colleagues in the CERN accelerator departments for the excellent performance of the LHC. We thank the technical and administrative staff at the LHCb institutes. We acknowledge support from CERN and from the national agencies: CAPES, CNPq, FAPERJ and FINEP (Brazil); MOST and NSFC (China); CNRS/ IN2P3 (France); BMBF, DFG and MPG (Germany); INFN (Italy); NWO (The Netherlands); MNiSW and NCN (Poland); MEN/IFA (Romania); MinES and FASO (Russia); MinECo (Spain); SNSF and SER (Switzerland); NASU (Ukraine); STFC (United Kingdom); NSF (USA). We acknowledge the computing resources that are provided by CERN, IN2P3 (France), KIT and DESY (Germany), INFN (Italy), SURF (The Netherlands), PIC (Spain), GridPP (United Kingdom), RRCKI and Yandex LLC (Russia), CSCS (Switzerland), IFIN-HH (Romania), CBPF (Brazil), PL-GRID (Poland) and OSC (USA). We are indebted to the communities behind the multiple open source software packages on which we depend. Individual groups or members have received support from $\mathrm{AvH}$ Foundation (Germany), EPLANET, Marie SkłodowskaCurie Actions and ERC (European Union), Conseil Général de Haute-Savoie, Labex ENIGMASS and OCEVU, Région Auvergne (France), RFBR and Yandex LLC (Russia), GVA, XuntaGal and GENCAT (Spain), Herchel Smith Fund, The Royal Society, Royal Commission for the Exhibition of 1851 and the Leverhulme Trust (United Kingdom).
[1] K. Abe et al. (Belle Collaboration), Study of $B^{-} \rightarrow$ $D^{* * 0} \pi^{-}\left(D^{* * 0} \rightarrow D^{(*)+} \pi^{-}\right)$decays, Phys. Rev. D 69, 112002 (2004).

[2] A. Kuzmin et al. (Belle Collaboration), Study of $\bar{B}^{0} \rightarrow$ $D^{0} \pi^{+} \pi^{-}$decays, Phys. Rev. D 76, 012006 (2007).

[3] B. Aubert et al. (BABAR Collaboration), Dalitz plot analysis of $B^{-} \rightarrow D^{+} \pi^{-} \pi^{-}$, Phys. Rev. D 79, 112004 (2009).
[4] P. del Amo Sanchez et al. (BABAR Collaboration), Dalitzplot analysis of $B^{0} \rightarrow \bar{D}^{0} \pi^{+} \pi^{-}$, arXiv:1007.4464.

[5] R. Aaij et al. (LHCb Collaboration), Dalitz plot analysis of $B^{0} \rightarrow \bar{D}^{0} \pi^{+} \pi^{-}$decays, Phys. Rev. D 92, 032002 (2015).

[6] R. Aaij et al. (LHCb Collaboration), Amplitude analysis of $B^{-} \rightarrow D^{+} \pi^{-} \pi^{-}$decays, Phys. Rev. D 94, 072001 (2016). 
[7] R. Aaij et al. (LHCb Collaboration), First observation and amplitude analysis of the $B^{-} \rightarrow D^{+} K^{-} \pi^{-}$decay, Phys. Rev. D 91, 092002 (2015); Erratum, Phys. Rev. D 93, 119901(E) (2016).

[8] R. Aaij et al. (LHCb Collaboration), Amplitude analysis of $B^{0} \rightarrow \bar{D}^{0} K^{+} \pi^{-}$decays, Phys. Rev. D 92, 012012 (2015).

[9] R. Aaij et al. (LHCb Collaboration), Observation of Overlapping Spin-1 and Spin-3 $\bar{D}^{0} K^{-}$Resonances at Mass $2.86 \mathrm{GeV} / c^{2}$, Phys. Rev. Lett. 113, 162001 (2014).

[10] R. Aaij et al. (LHCb Collaboration), Dalitz plot analysis of $B_{s}^{0} \rightarrow \bar{D}^{0} K^{-} \pi^{+}$decays, Phys. Rev. D 90, 072003 (2014).

[11] P. del Amo Sanchez et al. (BABAR Collaboration), Observation of new resonances decaying to $D \pi$ and $D^{*} \pi$ in inclusive $e^{+} e^{-}$collisions near $\sqrt{s}=10.58 \mathrm{GeV}$, Phys. Rev. D 82, 111101 (2010).

[12] R. Aaij et al. (LHCb Collaboration), Study of $D_{J}$ meson decays to $D^{+} \pi^{-}, D^{0} \pi^{+}$and $D^{*+} \pi^{-}$final states in $p p$ collisions, J. High Energy Phys. 09 (2013) 145.

[13] N. Cabibbo, Unitary Symmetry and Leptonic Decays, Phys. Rev. Lett. 10, 531 (1963).

[14] M. Kobayashi and T. Maskawa, $C P$ violation in the renormalizable theory of weak interaction, Prog. Theor. Phys. 49, 652 (1973).

[15] N. Sinha, Determining $\gamma$ using $B \rightarrow D^{* *} K$, Phys. Rev. D 70, 097501 (2004).

[16] R. Aaij et al. (LHCb Collaboration), First observation of the rare $B^{+} \rightarrow D^{+} K^{+} \pi^{-}$decay, Phys. Rev. D 93, 051101(R) (2016); Erratum, Phys. Rev. D 93, 119902(E) (2016).

[17] R. Aaij et al. (LHCb Collaboration), Measurement of the CKM angle $\gamma$ from a combination of LHCb results, J. High Energy Phys. 12 (2016) 087.

[18] A. A. Alves Jr. et al. (LHCb Collaboration), The LHCb detector at the LHC, J. Instrum. 3, S08005 (2008).

[19] R. Aaij et al. (LHCb Collaboration), LHCb detector performance, Int. J. Mod. Phys. A 30, 1530022 (2015).

[20] V. V. Gligorov and M. Williams, Efficient, reliable and fast high-level triggering using a bonsai boosted decision tree, J. Instrum. 8, P02013 (2013).

[21] R. Aaij et al., The LHCb trigger and its performance in 2011, J. Instrum. 8, P04022 (2013).

[22] T. Sjöstrand, S. Mrenna, and P. Skands, PYTHIA 6.4 physics and manual, J. High Energy Phys. 05 (2006) 026; A brief introduction to PYTHIA 8.1, Comput. Phys. Commun. 178, 852 (2008).
[23] I. Belyaev et al., Handling of the generation of primary events in Gauss, the LHCb simulation framework, J. Phys. Conf. Ser. 331, 032047 (2011).

[24] D. J. Lange, The EvtGen particle decay simulation package, Nucl. Instrum. Methods Phys. Res., Sect. A 462, 152 (2001).

[25] P. Golonka and Z. Was, PHOTOS Monte Carlo: A precision tool for QED corrections in $Z$ and $W$ decays, Eur. Phys. J. C 45, 97 (2006).

[26] J. Allison et al. (GEANT4 Collaboration), GEANT4 developments and applications, IEEE Trans. Nucl. Sci. 53, 270 (2006); S. Agostinelli et al. (GEANT4 Collaboration), GEANT4: A simulation toolkit, Nucl. Instrum. Methods Phys. Res., Sect. A 506, 250 (2003).

[27] M. Clemencic, G. Corti, S. Easo, C. R. Jones, S. Miglioranzi, M. Pappagallo, and P. Robbe, The LHCb simulation application, Gauss: Design, evolution and experience, J. Phys. Conf. Ser. 331, 032023 (2011).

[28] C. Patrignani et al. (Particle Data Group Collaboration), Review of particle physics, Chin. Phys. C 40, 100001 (2016).

[29] M. Feindt and U. Kerzel, The NeuroBayes neural network package, Nucl. Instrum. Methods Phys. Res., Sect. A 559, 190 (2006).

[30] G. Punzi, Sensitivity of searches for new signals and its optimization, 2003, edited by L. Lyons, R. Mount, and R. Reitmeyer, eConf C030908, p. 79 (2003).

[31] M. Adinolfi et al., Performance of the LHCb RICH detector at the LHC, Eur. Phys. J. C 73, 2431 (2013).

[32] T. Skwarnicki, PhD thesis, Institute of Nuclear Physics, Krakow, 1986, DESY-F31-86-02.

[33] H. Albrecht et al. (ARGUS Collaboration), Search for Hadronic $b \rightarrow u$ Decays, Phys. Lett. B 241, 278 (1990).

[34] R. Aaij et al. (LHCb Collaboration), Observations of $\Lambda_{b}^{0} \rightarrow$ $\Lambda K^{+} \pi^{-}$and $\Lambda_{b}^{0} \rightarrow \Lambda K^{+} K^{-}$decays and searches for other $\Lambda_{b}^{0}$ and $\Xi_{b}^{0}$ decays to $\Lambda h^{+} h^{-}$final states, J. High Energy Phys. 05 (2016) 081.

[35] R. Aaij et al. (LHCb Collaboration), Observation of the Annihilation Decay Mode $B^{0} \rightarrow K^{+} K^{-}$, Phys. Rev. Lett. 118, 081801 (2017).

[36] M. Pivk and F. R. Le Diberder, sPlot: A statistical tool to unfold data distributions, Nucl. Instrum. Methods Phys. Res., Sect. A 555, 356 (2005).

[37] R. Aaij et al. (LHCb Collaboration), Observation of $B^{0} \rightarrow$ $\bar{D}^{0} K^{+} K^{-}$and Evidence for $B_{s}^{0} \rightarrow \bar{D}^{0} K^{+} K^{-}$, Phys. Rev. Lett. 109, 131801 (2012).

R. Aaij, ${ }^{40}$ B. Adeva, ${ }^{39}$ M. Adinolfi, ${ }^{48}$ Z. Ajaltouni, ${ }^{5}$ S. Akar, ${ }^{59}$ J. Albrecht, ${ }^{10}$ F. Alessio, ${ }^{40}$ M. Alexander, ${ }^{53}$ S. Ali, ${ }^{43}$ G. Alkhazov, ${ }^{31}$ P. Alvarez Cartelle, ${ }^{55}$ A. A. Alves Jr., ${ }^{59}$ S. Amato, ${ }^{2}$ S. Amerio, ${ }^{23}$ Y. Amhis, ${ }^{7}$ L. An, ${ }^{3}$ L. Anderlini, ${ }^{18}$ G. Andreassi, ${ }^{41}$ M. Andreotti, ${ }^{17, g}$ J. E. Andrews, ${ }^{60}$ R. B. Appleby, ${ }^{56}$ F. Archilli, ${ }^{43}$ P. d'Argent, ${ }^{12}$ J. Arnau Romeu, ${ }^{6}$ A. Artamonov, ${ }^{37}$ M. Artuso, ${ }^{61}$ E. Aslanides, ${ }^{6}$ G. Auriemma, ${ }^{26}$ M. Baalouch, ${ }^{5}$ I. Babuschkin, ${ }^{56}$ S. Bachmann, ${ }^{12}$ J. J. Back, ${ }^{50}$ A. Badalov, ${ }^{38}$ C. Baesso, ${ }^{62}$ S. Baker, ${ }^{55}$ V. Balagura, ${ }^{7, c}$ W. Baldini, ${ }^{17}$ A. Baranov, ${ }^{35}$ R. J. Barlow, ${ }^{56}$ C. Barschel, ${ }^{40}$ S. Barsuk, ${ }^{7}$ W. Barter, ${ }^{56}$ F. Baryshnikov, ${ }^{32}$ M. Baszczyk, ${ }^{27}$ V. Batozskaya, ${ }^{29}$ V. Battista, ${ }^{41}$ A. Bay, ${ }^{41}$ L. Beaucourt, $^{4}$ J. Beddow, $^{53}$ F. Bedeschi, ${ }^{24}$ I. Bediaga, ${ }^{1}$ A. Beiter, ${ }^{61}$ L. J. Bel, ${ }^{43}$ V. Bellee, ${ }^{41}$ N. Belloli, ${ }^{21, i}$ K. Belous, ${ }^{37}$ I. Belyaev, $^{32}$ E. Ben-Haim, ${ }^{8}$ G. Bencivenni, ${ }^{19}$ S. Benson, ${ }^{43}$ S. Beranek, ${ }^{9}$ A. Berezhnoy, ${ }^{33}$ R. Bernet, ${ }^{42}$ A. Bertolin, ${ }^{23}$ C. Betancourt, ${ }^{42}$ F. Betti, ${ }^{15}$ M.-O. Bettler, ${ }^{40}$ M. van Beuzekom, ${ }^{43}$ Ia. Bezshyiko, ${ }^{42}$ S. Bifani, ${ }^{47}$ P. Billoir, ${ }^{8}$ A. Birnkraut, ${ }^{10}$ A. Bitadze, ${ }^{56}$ A. Bizzeti, ${ }^{18, u}$ 
T. Blake, ${ }^{50}$ F. Blanc, ${ }^{41}$ J. Blouw,,${ }^{11 \dagger}$ S. Blusk,${ }^{61}$ V. Bocci, ${ }^{26}$ T. Boettcher, ${ }^{58}$ A. Bondar, ${ }^{36, w}$ N. Bondar, ${ }^{31}$ W. Bonivento, ${ }^{16}$ I. Bordyuzhin, ${ }^{32}$ A. Borgheresi, ${ }^{21, i}$ S. Borghi, ${ }^{56}$ M. Borisyak,${ }^{35}$ M. Borsato, ${ }^{39}$ F. Bossu, ${ }^{7}$ M. Boubdir, ${ }^{9}$ T. J. V. Bowcock, ${ }^{54}$ E. Bowen, ${ }^{42}$ C. Bozzi,${ }^{17}{ }^{40}$ S. Braun, ${ }^{12}$ T. Britton, ${ }^{61}$ J. Brodzicka,${ }^{56}$ E. Buchanan, ${ }^{48}$ C. Burr, ${ }^{56}$ A. Bursche, ${ }^{2}$ J. Buytaert,${ }^{40}$ S. Cadeddu, ${ }^{16}$ R. Calabrese, ${ }^{17, g}$ M. Calvi, ${ }^{21, i}$ M. Calvo Gomez, ${ }^{38, m}$ A. Camboni, ${ }^{38}$ P. Campana, ${ }^{19}$ D. H. Campora Perez, ${ }^{40}$ L. Capriotti, ${ }^{56}$ A. Carbone, ${ }^{15, \mathrm{e}}$ G. Carboni, ${ }^{25, \mathrm{j}}$ R. Cardinale, ${ }^{20, \mathrm{~h}}$ A. Cardini, ${ }^{16}$ P. Carniti, ${ }^{21, \mathrm{i}}$ L. Carson, ${ }^{52}$ K. Carvalho Akiba, ${ }^{2}$ G. Casse ${ }^{54}$ L. Cassina, ${ }^{21, i}$ L. Castillo Garcia, ${ }^{41}$ M. Cattaneo,${ }^{40}$ G. Cavallero, ${ }^{20}$ R. Cenci, ${ }^{24, t}$ D. Chamont, ${ }^{7}$ M. Charles, ${ }^{8}$ Ph. Charpentier, ${ }^{40}$ G. Chatzikonstantinidis, ${ }^{47}$ M. Chefdeville, ${ }^{4}$ S. Chen, ${ }^{56}$ S.-F. Cheung, ${ }^{57}$ V. Chobanova,${ }^{39}$ M. Chrzaszcz, ${ }^{42,27}$ A. Chubykin, ${ }^{31}$ X. Cid Vidal, ${ }^{39}$ G. Ciezarek, ${ }^{43}$ P. E. L. Clarke, ${ }^{52}$ M. Clemencic, ${ }^{40}$ H. V. Cliff, ${ }^{49}$ J. Closier, ${ }^{40}$ V. Coco, ${ }^{59}$ J. Cogan, ${ }^{6}$ E. Cogneras, ${ }^{5}$ V. Cogoni, ${ }^{16, f}$ L. Cojocariu, ${ }^{30}$ P. Collins, ${ }^{40}$ A. Comerma-Montells, ${ }^{12}$ A. Contu, ${ }^{40}$ A. Cook,${ }^{48}$ G. Coombs, ${ }^{40}$ S. Coquereau, ${ }^{38}$ G. Corti, ${ }^{40}$ M. Corvo, ${ }^{17, g}$ C. M. Costa Sobral, ${ }^{50}$ B. Couturier, ${ }^{40}$ G. A. Cowan, ${ }^{52}$ D. C. Craik, ${ }^{52}$ A. Crocombe, ${ }^{50}$ M. Cruz Torres,${ }^{62}$ S. Cunliffe, ${ }^{55}$ R. Currie, ${ }^{52}$ C. D'Ambrosio, ${ }^{40}$ F. Da Cunha Marinho, ${ }^{2}$ E. Dall'Occo, ${ }^{43}$ J. Dalseno, ${ }^{48}$ A. Davis, ${ }^{3}$ K. De Bruyn,${ }^{6}$ S. De Capua ${ }^{56}$ M. De Cian, ${ }^{12}$ J. M. De Miranda, ${ }^{1}$ L. De Paula, ${ }^{2}$ M. De Serio,${ }^{14, d}$ P. De Simone, ${ }^{19}$ C. T. Dean,${ }^{53}$ D. Decamp, ${ }^{4}$ M. Deckenhoff, ${ }^{10}$ L. Del Buono, ${ }^{8}$ H.-P. Dembinski, ${ }^{11}$ M. Demmer, ${ }^{10}$ A. Dendek,${ }^{28}$ D. Derkach, ${ }^{35}$ O. Deschamps, ${ }^{5}$ F. Dettori, ${ }^{54}$ B. Dey, ${ }^{22}$ A. Di Canto, ${ }^{40}$ P. Di Nezza, ${ }^{19}$ H. Dijkstra, ${ }^{40}$ F. Dordei, ${ }^{40}$ M. Dorigo, ${ }^{41}$ A. Dosil Suárez, ${ }^{39}$ A. Dovbnya,${ }^{45}$ K. Dreimanis,${ }^{54}$ L. Dufour, ${ }^{43}$ G. Dujany, ${ }^{56}$ K. Dungs,${ }^{40}$ P. Durante, ${ }^{40}$ R. Dzhelyadin, ${ }^{37}$ M. Dziewiecki, ${ }^{12}$ A. Dziurda, ${ }^{40}$ A. Dzyuba, ${ }^{31}$ N. Déléage, ${ }^{4}$ S. Easo, ${ }^{51}$ M. Ebert,${ }^{52}$ U. Egede, ${ }^{55}$ V. Egorychev, ${ }^{32}$ S. Eidelman, ${ }^{36, w}$ S. Eisenhardt, ${ }^{52}$ U. Eitschberger, ${ }^{10}$ R. Ekelhof ${ }^{10}$ L. Eklund, ${ }^{53}$ S. Ely, ${ }^{61}$ S. Esen, ${ }^{12}$ H. M. Evans, ${ }^{49}$ T. Evans, ${ }^{57}$ A. Falabella, ${ }^{15}$ N. Farley, ${ }^{47}$ S. Farry, ${ }^{54}$ R. Fay, ${ }^{54}$ D. Fazzini, ${ }^{21, i}$ D. Ferguson, ${ }^{52}$ G. Fernandez,${ }^{38}$ A. Fernandez Prieto,${ }^{39}$ F. Ferrari, ${ }^{15}$ F. Ferreira Rodrigues, ${ }^{2}$ M. Ferro-Luzzi, ${ }^{40}$ S. Filippov, ${ }^{34}$ R. A. Fini, ${ }^{14}$ M. Fiore, ${ }^{17, g}$ M. Fiorini, ${ }^{17, g}$ M. Firlej, ${ }^{28}$ C. Fitzpatrick,${ }^{41}$ T. Fiutowski, ${ }^{28}$ F. Fleuret, ${ }^{7, b}$ K. Fohl, ${ }^{40}$ M. Fontana, ${ }^{16,40}$ F. Fontanelli, ${ }^{20, h}$ D. C. Forshaw ${ }^{61}$ R. Forty,${ }^{40}$ V. Franco Lima, ${ }^{54}$ M. Frank, ${ }^{40}$ C. Frei, ${ }^{40}$ J. Fu, ${ }^{22, q}$ W. Funk,${ }^{40}$ E. Furfaro, ${ }^{25, j}$ C. Färber, ${ }^{40}$ A. Gallas Torreira ${ }^{39}$ D. Galli, ${ }^{15, \mathrm{e}}$ S. Gallorini ${ }^{23}$ S. Gambetta, ${ }^{52}$ M. Gandelman, ${ }^{2}$ P. Gandini, ${ }^{57}$ Y. Gao, ${ }^{3}$ L. M. Garcia Martin, ${ }^{69}$ J. García Pardiñas, ${ }^{39}$ J. Garra Tico, ${ }^{49}$ L. Garrido,${ }^{38}$ P. J. Garsed,${ }^{49}$ D. Gascon, ${ }^{38}$ C. Gaspar, ${ }^{40}$ L. Gavardi, ${ }^{10}$ G. Gazzoni, ${ }^{5}$ D. Gerick, ${ }^{12}$ E. Gersabeck, ${ }^{12}$ M. Gersabeck, ${ }^{56}$ T. Gershon,${ }^{50}$ Ph. Ghez,${ }^{4}$ S. Gianì, ${ }^{41}$ V. Gibson, ${ }^{49}$ O. G. Girard, ${ }^{41}$ L. Giubega, ${ }^{30}$ K. Gizdov, ${ }^{52}$ V. V. Gligorov, ${ }^{8}$ D. Golubkov, ${ }^{32}$ A. Golutvin, ${ }^{55,40}$ A. Gomes, ${ }^{1, a}$ I. V. Gorelov, ${ }^{33}$ C. Gotti, ${ }^{21, i}$ E. Govorkova, ${ }^{43}$ R. Graciani Diaz,${ }^{38}$ L. A. Granado Cardoso,${ }^{40}$ E. Graugés, ${ }^{38}$ E. Graverini, ${ }^{42}$ G. Graziani, ${ }^{18}$ A. Grecu, ${ }^{30}$ R. Greim, ${ }^{9}$ P. Griffith, ${ }^{16}$ L. Grillo, ${ }^{21,40, i}$ B. R. Gruberg Cazon,${ }^{57}$ O. Grünberg, ${ }^{67}$ E. Gushchin, ${ }^{34}$ Yu. Guz, ${ }^{37}$ T. Gys, ${ }^{40}$ C. Göbel, ${ }^{62}$ T. Hadavizadeh,${ }^{57}$ C. Hadjivasiliou, ${ }^{5}$ G. Haefeli, ${ }^{41}$ C. Haen,${ }^{40}$ S. C. Haines,${ }^{49}$ B. Hamilton, ${ }^{60}$ X. Han,${ }^{12}$ S. Hansmann-Menzemer, ${ }^{12}$ N. Harnew, ${ }^{57}$ S. T. Harnew, ${ }^{48}$ J. Harrison, ${ }^{56}$ M. Hatch, ${ }^{40}$ J. He, ${ }^{63}$ T. Head, ${ }^{41}$ A. Heister, ${ }^{9}$ K. Hennessy, ${ }^{54}$ P. Henrard, ${ }^{5}$ L. Henry, ${ }^{69}$ E. van Herwijnen, ${ }^{40}$ M. Heß,${ }^{67}$ A. Hicheur, ${ }^{2}$ D. Hill,${ }^{57}$ C. Hombach, ${ }^{56}$ H. Hopchev, ${ }^{41}$ Z.-C. Huard, ${ }^{59}$ W. Hulsbergen, ${ }^{43}$ T. Humair, ${ }^{55}$ M. Hushchyn, ${ }^{35}$ D. Hutchcroft, ${ }^{54}$ M. Idzik, ${ }^{28}$ P. Ilten, ${ }^{58}$ R. Jacobsson, ${ }^{40}$ J. Jalocha, ${ }^{57}$ E. Jans, ${ }^{43}$ A. Jawahery, ${ }^{60}$ F. Jiang, ${ }^{3}$ M. John, ${ }^{57}$ D. Johnson, ${ }^{40}$ C. R. Jones, ${ }^{49}$ C. Joram, ${ }^{40}$ B. Jost, ${ }^{40}$ N. Jurik, ${ }^{57}$ S. Kandybei, ${ }^{45}$ M. Karacson, ${ }^{40}$ J. M. Kariuki, ${ }^{48}$ S. Karodia, ${ }^{53}$ M. Kecke, ${ }^{12}$ M. Kelsey, ${ }^{61}$ M. Kenzie, ${ }^{49}$ T. Ketel, ${ }^{44}$

E. Khairullin, ${ }^{35}$ B. Khanji, ${ }^{12}$ C. Khurewathanakul, ${ }^{41}$ T. Kirn, ${ }^{9}$ S. Klaver, ${ }^{56}$ K. Klimaszewski, ${ }^{29}$ T. Klimkovich, ${ }^{11}$ S. Koliiev, ${ }^{46}$ M. Kolpin, ${ }^{12}$ I. Komarov, ${ }^{41}$ R. Kopecna, ${ }^{12}$ P. Koppenburg, ${ }^{43}$ A. Kosmyntseva, ${ }^{32}$ S. Kotriakhova, ${ }^{31}$ A. Kozachuk, ${ }^{33}$ M. Kozeiha, ${ }^{5}$ L. Kravchuk, ${ }^{34}$ M. Kreps, ${ }^{50}$ P. Krokovny, ${ }^{36, w}$ F. Kruse, ${ }^{10}$ W. Krzemien, ${ }^{29}$ W. Kucewicz, ${ }^{27,1}$ M. Kucharczyk, ${ }^{27}$ V. Kudryavtsev, ${ }^{36, w}$ A. K. Kuonen, ${ }^{41}$ K. Kurek, ${ }^{29}$ T. Kvaratskheliya, ${ }^{32,40}$ D. Lacarrere, ${ }^{40}$ G. Lafferty, ${ }^{56}$ A. Lai, ${ }^{16}$ G. Lanfranchi, ${ }^{19}$ C. Langenbruch, ${ }^{9}$ T. Latham, ${ }^{50}$ C. Lazzeroni, ${ }^{47}$ R. Le Gac, ${ }^{6}$ J. van Leerdam, ${ }^{43}$ A. Leflat,${ }^{33,40}$ J. Lefrançois, ${ }^{7}$ R. Lefèvre, ${ }^{5}$ F. Lemaitre, ${ }^{40}$ E. Lemos Cid ${ }^{39}$ O. Leroy, ${ }^{6}$ T. Lesiak, ${ }^{27}$ B. Leverington, ${ }^{12} \mathrm{~T}_{\text {. Li }}{ }^{3}$ Y. Li, ${ }^{7} \mathrm{Z} . \mathrm{Li},{ }^{61}$ T. Likhomanenko, ${ }^{35,68}$ R. Lindner, ${ }^{40}$ F. Lionetto, ${ }^{42}$ X. Liu, ${ }^{3}$ D. Loh ${ }^{50}$ I. Longstaff, ${ }^{53}$ J. H. Lopes, ${ }^{2}$ D. Lucchesi, ${ }^{23,0}$ M. Lucio Martinez, ${ }^{39}$ H. Luo, ${ }^{52}$ A. Lupato, ${ }^{23}$ E. Luppi,${ }^{17, g}$ O. Lupton, ${ }^{40}$ A. Lusiani, ${ }^{24}$ X. Lyu, ${ }^{63}$ F. Machefert, ${ }^{7}$ F. Maciuc, ${ }^{30}$ O. Maev,${ }^{31}$ K. Maguire, ${ }^{56}$ S. Malde,${ }^{57}$ A. Malinin, ${ }^{68}$ T. Maltsev,${ }^{36}$ G. Manca, ${ }^{16, f}$ G. Mancinelli, ${ }^{6}$ P. Manning, ${ }^{61}$ J. Maratas, ${ }^{5, v}$ J. F. Marchand, ${ }^{4}$ U. Marconi, ${ }^{15}$ C. Marin Benito, ${ }^{38}$ M. Marinangeli, ${ }^{41}$ P. Marino, ${ }^{24, t}$ J. Marks, ${ }^{12}$ G. Martellotti, ${ }^{26}$ M. Martin, ${ }^{6}$ M. Martinelli, ${ }^{41}$ D. Martinez Santos ${ }^{39}$ F. Martinez Vidal, ${ }^{69}$ D. Martins Tostes, ${ }^{2}$ L. M. Massacrier, ${ }^{7}$ A. Massafferri, ${ }^{1}$

R. Matev ${ }^{40}$ A. Mathad ${ }^{50}$ Z. Mathe,${ }^{40}$ C. Matteuzzi, ${ }^{21}$ A. Mauri, ${ }^{42}$ E. Maurice, ${ }^{7, b}$ B. Maurin, ${ }^{41}$ A. Mazurov,${ }^{47}$ M. McCann, ${ }^{55,40}$ A. McNab,${ }^{56}$ R. McNulty, ${ }^{13}$ B. Meadows,${ }^{59}$ F. Meier,${ }^{10}$ D. Melnychuk, ${ }^{29}$ M. Merk, ${ }^{43}$ A. Merli, ${ }^{22,40, q}$ E. Michielin, ${ }^{23}$ D. A. Milanes, ${ }^{66}$ M.-N. Minard, ${ }^{4}$ D. S. Mitzel, ${ }^{12}$ A. Mogini, ${ }^{8}$ J. Molina Rodriguez, ${ }^{1}$ I. A. Monroy, ${ }^{66}$ S. Monteil, ${ }^{5}$ M. Morandin, ${ }^{23}$ M. J. Morello, ${ }^{24, t}$ O. Morgunova, ${ }^{68}$ J. Moron, ${ }^{28}$ A. B. Morris,${ }^{52}$ R. Mountain, ${ }^{61}$ F. Muheim, ${ }^{52}$ M. Mulder, ${ }^{43}$ M. Mussini, ${ }^{15}$ D. Müller, ${ }^{56}$ J. Müller, ${ }^{10}$ K. Müller, ${ }^{42}$ V. Müller, ${ }^{10}$ P. Naik, ${ }^{48}$ T. Nakada, ${ }^{41}$ R. Nandakumar, ${ }^{51}$ 
A. Nandi, ${ }^{57}$ I. Nasteva, ${ }^{2}$ M. Needham, ${ }^{52}$ N. Neri, ${ }^{22,40}$ S. Neubert, ${ }^{12}$ N. Neufeld, ${ }^{40}$ M. Neuner, ${ }^{12}$ T. D. Nguyen, ${ }^{41}$ C. Nguyen-Mau, ${ }^{41, \mathrm{n}}$ S. Nieswand, ${ }^{9}$ R. Niet, ${ }^{10}$ N. Nikitin, ${ }^{33}$ T. Nikodem, ${ }^{12}$ A. Nogay, ${ }^{68}$ A. Novoselov, ${ }^{37}$ D. P. O'Hanlon, ${ }^{50}$

A. Oblakowska-Mucha, ${ }^{28}$ V. Obraztsov, ${ }^{37}$ S. Ogilvy, ${ }^{19}$ R. Oldeman, ${ }^{16, f}$ C. J. G. Onderwater, ${ }^{70}$ A. Ossowska, ${ }^{27}$ J. M. Otalora Goicochea, ${ }^{2}$ P. Owen, ${ }^{42}$ A. Oyanguren, ${ }^{69}$ P. R. Pais, ${ }^{41}$ A. Palano, ${ }^{14, d}$ M. Palutan, ${ }^{19,40}$ A. Papanestis, ${ }^{51}$ M. Pappagallo, ${ }^{14, \mathrm{~d}}$ L. L. Pappalardo, ${ }^{17, g}$ C. Pappenheimer,${ }^{59}$ W. Parker,${ }^{60}$ C. Parkes,${ }^{56}$ G. Passaleva, ${ }^{18}$ A. Pastore, ${ }^{14, d}$ M. Patel,${ }^{55}$ C. Patrignani, ${ }^{15, e}$ A. Pearce, ${ }^{40}$ A. Pellegrino, ${ }^{43}$ G. Penso,${ }^{26}$ M. Pepe Altarelli, ${ }^{40}$ S. Perazzini,${ }^{40}$ P. Perret, ${ }^{5}$ L. Pescatore, ${ }^{41}$ K. Petridis, ${ }^{48}$ A. Petrolini,${ }^{20, h}$ A. Petrov,${ }^{68}$ M. Petruzzo, ${ }^{22, q}$ E. Picatoste Olloqui, ${ }^{38}$ B. Pietrzyk,${ }^{4}$ M. Pikies,${ }^{27}$ D. Pinci, ${ }^{26}$ A. Pistone,${ }^{20}$ A. Piucci, ${ }^{12}$ V. Placinta,${ }^{30}$ S. Playfer,${ }^{52}$ M. Plo Casasus, ${ }^{39}$ T. Poikela, ${ }^{40}$ F. Polci, ${ }^{8}$ M. Poli Lener, ${ }^{19}$ A. Poluektov, ${ }^{50,36}$ I. Polyakov, ${ }^{61}$ E. Polycarpo, ${ }^{2}$ G. J. Pomery, ${ }^{48}$ S. Ponce,${ }^{40}$ A. Popov, ${ }^{37}$ D. Popov, ${ }^{11,40}$ B. Popovici, ${ }^{30}$ S. Poslavskii, ${ }^{37}$ C. Potterat, ${ }^{2}$ E. Price,${ }^{48}$ J. Prisciandaro, ${ }^{39}$ C. Prouve, ${ }^{48}$ V. Pugatch,${ }^{46}$ A. Puig Navarro, ${ }^{42}$ G. Punzi, ${ }^{24, p}$ C. Qian, ${ }^{63}$ W. Qian, ${ }^{50}$ R. Quagliani, ${ }^{7,48}$ B. Rachwal, ${ }^{28}$ J. H. Rademacker, ${ }^{48}$ M. Rama, ${ }^{24}$ M. Ramos Pernas,${ }^{39}$ M. S. Rangel, ${ }^{2}$ I. Raniuk, ${ }^{45,}$ F. Ratnikov, ${ }^{35}$ G. Raven,${ }^{44}$ F. Redi,${ }^{55}$ S. Reichert, ${ }^{10}$ A. C. dos Reis, ${ }^{1}$ C. Remon Alepuz, ${ }^{69}$ V. Renaudin, ${ }^{7}$ R. P. Rera, ${ }^{50}$ S. Ricciardi, ${ }^{51}$ S. Richards, ${ }^{48}$ M. Rihl,${ }^{40}$ K. Rinnert,${ }^{54}$ V. Rives Molina, ${ }^{38}$ P. Robbe, ${ }^{7}$ A. B. Rodrigues, ${ }^{1}$ E. Rodrigues, ${ }^{59}$ J. A. Rodriguez Lopez, ${ }^{66}$ P. Rodriguez Perez, ${ }^{56,}$ A. Rogozhnikov, ${ }^{35}$ S. Roiser, ${ }^{40}$ A. Rollings,${ }^{57}$ V. Romanovskiy, ${ }^{37}$ A. Romero Vidal,${ }^{39}$ J. W. Ronayne, ${ }^{13}$ M. Rotondo, ${ }^{19}$ M. S. Rudolph, ${ }^{61}$ T. Ruf, ${ }^{40}$ P. Ruiz Valls, ${ }^{69}$ J. J. Saborido Silva, ${ }^{39}$ E. Sadykhov, ${ }^{32}$ N. Sagidova, ${ }^{31}$ B. Saitta, ${ }^{16, f}$ V. Salustino Guimaraes, ${ }^{1}$ D. Sanchez Gonzalo, ${ }^{38}$ C. Sanchez Mayordomo, ${ }^{69}$ B. Sanmartin Sedes,${ }^{39}$ R. Santacesaria, ${ }^{26}$ C. Santamarina Rios, ${ }^{39}$ M. Santimaria, ${ }^{19}$ E. Santovetti, ${ }^{25, j}$ A. Sarti, ${ }^{19, k}$ C. Satriano, ${ }^{26, s}$ A. Satta, ${ }^{25}$ D. M. Saunders, ${ }^{48}$ D. Savrina, ${ }^{32,33}$ S. Schael, ${ }^{9}$ M. Schellenberg, ${ }^{10}$ M. Schiller, ${ }^{53}$ H. Schindler, ${ }^{40}$ M. Schlupp, ${ }^{10}$ M. Schmelling, ${ }^{11}$ T. Schmelzer, ${ }^{10}$ B. Schmidt,${ }^{40}$ O. Schneider, ${ }^{41}$ A. Schopper, ${ }^{40}$ H. F. Schreiner, ${ }^{59}$ K. Schubert, ${ }^{10}$ M. Schubiger,${ }^{41}$ M.-H. Schune, ${ }^{7}$ R. Schwemmer, ${ }^{40}$ B. Sciascia, ${ }^{19}$ A. Sciubba, ${ }^{26, k}$ A. Semennikov, ${ }^{32}$ A. Sergi,${ }^{47}$ N. Serra, ${ }^{42}$ J. Serrano, ${ }^{6}$ L. Sestini,${ }^{23}$ P. Seyfert, ${ }^{21}$ M. Shapkin, ${ }^{37}$ I. Shapoval, ${ }^{45}$ Y. Shcheglov, ${ }^{31}$ T. Shears, ${ }^{54}$ L. Shekhtman, ${ }^{36, w}$ V. Shevchenko, ${ }^{68}$ B. G. Siddi, ${ }^{17,40}$ R. Silva Coutinho, ${ }^{42}$ L. Silva de Oliveira, ${ }^{2}$ G. Simi, ${ }^{23,0}$ S. Simone, ${ }^{14, d}$ M. Sirendi, ${ }^{49}$ N. Skidmore, ${ }^{48}$ T. Skwarnicki, ${ }^{61}$ E. Smith, ${ }^{55}$ I. T. Smith,${ }^{52}$ J. Smith,${ }^{49}$ M. Smith, ${ }^{55}$ 1. Soares Lavra, ${ }^{1}$ M. D. Sokoloff, ${ }^{59}$ F. J. P. Soler,${ }^{53}$ B. Souza De Paula, ${ }^{2}$ B. Spaan, ${ }^{10}$ P. Spradlin, ${ }^{53}$ S. Sridharan, ${ }^{40}$ F. Stagni, ${ }^{40}$ M. Stahl, ${ }^{12}$ S. Stahl, ${ }^{40}$ P. Stefko, ${ }^{41}$ S. Stefkova, ${ }^{55}$ O. Steinkamp, ${ }^{42}$ S. Stemmle, ${ }^{12}$ O. Stenyakin, ${ }^{37}$ H. Stevens,${ }^{10}$ S. Stoica,${ }^{30}$ S. Stone, ${ }^{61}$ B. Storaci, ${ }^{42}$ S. Stracka, ${ }^{24, p}$ M. E. Stramaglia, ${ }^{41}$ M. Straticiuc,${ }^{30}$ U. Straumann, ${ }^{42}$ L. Sun, ${ }^{64}$ W. Sutcliffe, ${ }^{55}$ K. Swientek, ${ }^{28}$ V. Syropoulos, ${ }^{44}$ M. Szczekowski, ${ }^{29}$ T. Szumlak, ${ }^{28}$ S. T'Jampens, ${ }^{4}$ A. Tayduganov, ${ }^{6}$ T. Tekampe, ${ }^{10}$ G. Tellarini, ${ }^{17, g}$ F. Teubert, ${ }^{40}$ E. Thomas, ${ }^{40}$ J. van Tilburg, ${ }^{43}$ M. J. Tilley,${ }^{55}$ V. Tisserand, ${ }^{4}$ M. Tobin, ${ }^{41}$ S. Tolk, ${ }^{49}$ L. Tomassetti, ${ }^{17, g}$ D. Tonelli, ${ }^{24}$ S. Topp-Joergensen,${ }^{57}$ F. Toriello, ${ }^{61}$ R. Tourinho Jadallah Aoude, ${ }^{1}$ E. Tournefier,${ }^{4}$ S. Tourneur, ${ }^{41}$ K. Trabelsi, ${ }^{41}$ M. Traill,${ }^{53}$ M. T. Tran,${ }^{41}$ M. Tresch, ${ }^{42}$ A. Trisovic, ${ }^{40}$ A. Tsaregorodtsev, ${ }^{6}$ P. Tsopelas, ${ }^{43}$ A. Tully, ${ }^{49}$ N. Tuning, ${ }^{43}$ A. Ukleja, ${ }^{29}$ A. Ustyuzhanin, ${ }^{35}$ U. Uwer, ${ }^{12}$ C. Vacca, ${ }^{16, f}$ V. Vagnoni, ${ }^{15,40}$ A. Valassi, ${ }^{40}$ S. Valat ${ }^{40}$ G. Valenti, ${ }^{15}$ R. Vazquez Gomez, ${ }^{19}$ P. Vazquez Regueiro, ${ }^{39}$ S. Vecchi, ${ }^{17}$ M. van Veghel, ${ }^{43}$ J. J. Velthuis, ${ }^{48}$ M. Veltri, ${ }^{18, r}$ G. Veneziano, ${ }^{57}$ A. Venkateswaran, ${ }^{61}$ T. A. Verlage, ${ }^{9}$ M. Vernet, ${ }^{5}$ M. Vesterinen, ${ }^{12}$ J. V. Viana Barbosa, ${ }^{40}$ B. Viaud, ${ }^{7}$ D. Vieira, ${ }^{63}$ M. Vieites Diaz, ${ }^{39}$ H. Viemann, ${ }^{67}$ X. Vilasis-Cardona, ${ }^{38, m}$ M. Vitti, ${ }^{49}$ V. Volkov, ${ }^{33}$ A. Vollhardt,${ }^{42}$ B. Voneki, ${ }^{40}$ A. Vorobyev ${ }^{31}$ V. Vorobyev, ${ }^{36, w}$ C. Voß,${ }^{9}$ J. A. de Vries, ${ }^{43}$ C. Vázquez Sierra ${ }^{39}$ R. Waldi, ${ }^{67}$ C. Wallace,${ }^{50}$ R. Wallace, ${ }^{13}$ J. Walsh, ${ }^{24}$ J. Wang, ${ }^{61}$ D. R. Ward ${ }^{49}$ H. M. Wark, ${ }^{54}$ N. K. Watson, ${ }^{47}$ D. Websdale ${ }^{55}$ A. Weiden, ${ }^{42}$ M. Whitehead, ${ }^{40}$ J. Wicht ${ }^{50}$ G. Wilkinson, ${ }^{57,40}$ M. Wilkinson, ${ }^{61}$ M. Williams, ${ }^{40}$ M. P. Williams, ${ }^{47}$ M. Williams, ${ }^{58}$ T. Williams, ${ }^{47}$ F. F. Wilson, ${ }^{51}$ J. Wimberley, ${ }^{60}$ M. A. Winn, ${ }^{7}$ J. Wishahi, ${ }^{10}$ W. Wislicki, ${ }^{29}$ M. Witek, ${ }^{27}$ G. Wormser, ${ }^{7}$ S. A. Wotton, ${ }^{49}$ K. Wraight,${ }^{53}$ K. Wyllie, ${ }^{40}$ Y. Xie, ${ }^{65}$ Z. Xu, ${ }^{4}$ Z. Yang, ${ }^{3}$ Z. Yang, ${ }^{60}$ Y. Yao,${ }^{61}$ H. Yin, ${ }^{65}$ J. Yu, ${ }^{65}$ X. Yuan, ${ }^{36, w}$

O. Yushchenko, ${ }^{37}$ K. A. Zarebski, ${ }^{47}$ M. Zavertyaev, ${ }^{11, \mathrm{c}}$ L. Zhang, ${ }^{3}$ Y. Zhang, ${ }^{7}$ A. Zhelezov, ${ }^{12}$ Y. Zheng, ${ }^{63}$ X. Zhu, ${ }^{3}$ V. Zhukov, ${ }^{33}$ and S. Zucchelli ${ }^{15}$

(LHCb Collaboration)

\author{
${ }^{1}$ Centro Brasileiro de Pesquisas Físicas (CBPF), Rio de Janeiro, Brazil \\ ${ }^{2}$ Universidade Federal do Rio de Janeiro (UFRJ), Rio de Janeiro, Brazil \\ ${ }^{3}$ Center for High Energy Physics, Tsinghua University, Beijing, China \\ ${ }^{4}$ LAPP, Université Savoie Mont-Blanc, CNRS/IN2P3, Annecy-Le-Vieux, France \\ ${ }^{5}$ Clermont Université, Université Blaise Pascal, CNRS/IN2P3, LPC, Clermont-Ferrand, France \\ ${ }^{6} \mathrm{CPPM}$, Aix-Marseille Université, CNRS/IN2P3, Marseille, France
}


${ }^{7}$ LAL, Université Paris-Sud, CNRS/IN2P3, Orsay, France

${ }^{8}$ LPNHE, Université Pierre et Marie Curie, Université Paris Diderot, CNRS/IN2P3, Paris, France

${ }^{9}$ I. Physikalisches Institut, RWTH Aachen University, Aachen, Germany

${ }^{10}$ Fakultät Physik, Technische Universität Dortmund, Dortmund, Germany

${ }^{11}$ Max-Planck-Institut für Kernphysik (MPIK), Heidelberg, Germany

${ }^{12}$ Physikalisches Institut, Ruprecht-Karls-Universität Heidelberg, Heidelberg, Germany

${ }^{13}$ School of Physics, University College Dublin, Dublin, Ireland

${ }^{14}$ Sezione INFN di Bari, Bari, Italy

${ }^{15}$ Sezione INFN di Bologna, Bologna, Italy

${ }^{16}$ Sezione INFN di Cagliari, Cagliari, Italy

${ }^{17}$ Sezione INFN di Ferrara, Ferrara, Italy

${ }^{18}$ Sezione INFN di Firenze, Firenze, Italy

${ }^{19}$ Laboratori Nazionali dell'INFN di Frascati, Frascati, Italy

${ }^{20}$ Sezione INFN di Genova, Genova, Italy

${ }^{21}$ Sezione INFN di Milano Bicocca, Milano, Italy

${ }^{22}$ Sezione INFN di Milano, Milano, Italy

${ }^{23}$ Sezione INFN di Padova, Padova, Italy

${ }^{24}$ Sezione INFN di Pisa, Pisa, Italy

${ }^{25}$ Sezione INFN di Roma Tor Vergata, Roma, Italy

${ }^{26}$ Sezione INFN di Roma La Sapienza, Roma, Italy

${ }^{27}$ Henryk Niewodniczanski Institute of Nuclear Physics Polish Academy of Sciences, Kraków, Poland

${ }^{28}$ AGH-University of Science and Technology, Faculty of Physics and Applied Computer Science, Kraków, Poland

${ }^{29}$ National Center for Nuclear Research (NCBJ), Warsaw, Poland

${ }^{30}$ Horia Hulubei National Institute of Physics and Nuclear Engineering, Bucharest-Magurele, Romania

${ }^{31}$ Petersburg Nuclear Physics Institute (PNPI), Gatchina, Russia

${ }^{32}$ Institute of Theoretical and Experimental Physics (ITEP), Moscow, Russia

${ }^{33}$ Institute of Nuclear Physics, Moscow State University (SINP MSU), Moscow, Russia

${ }^{34}$ Institute for Nuclear Research of the Russian Academy of Sciences (INR RAN), Moscow, Russia

${ }^{35}$ Yandex School of Data Analysis, Moscow, Russia

${ }^{36}$ Budker Institute of Nuclear Physics (SB RAS), Novosibirsk, Russia

${ }^{37}$ Institute for High Energy Physics (IHEP), Protvino, Russia

${ }^{38}$ ICCUB, Universitat de Barcelona, Barcelona, Spain

${ }^{39}$ Universidad de Santiago de Compostela, Santiago de Compostela, Spain

${ }^{40}$ European Organization for Nuclear Research (CERN), Geneva, Switzerland

${ }^{41}$ Institute of Physics, Ecole Polytechnique Fédérale de Lausanne (EPFL), Lausanne, Switzerland

${ }^{42}$ Physik-Institut, Universität Zürich, Zürich, Switzerland

${ }^{43}$ Nikhef National Institute for Subatomic Physics, Amsterdam, Netherlands

${ }^{44}$ Nikhef National Institute for Subatomic Physics and VU University Amsterdam, Amsterdam, Netherlands

${ }^{45}$ NSC Kharkiv Institute of Physics and Technology (NSC KIPT), Kharkiv, Ukraine

${ }^{46}$ Institute for Nuclear Research of the National Academy of Sciences (KINR), Kyiv, Ukraine

${ }^{47}$ University of Birmingham, Birmingham, United Kingdom

${ }^{48}$ H. H. Wills Physics Laboratory, University of Bristol, Bristol, United Kingdom

${ }^{49}$ Cavendish Laboratory, University of Cambridge, Cambridge, United Kingdom

${ }^{50}$ Department of Physics, University of Warwick, Coventry, United Kingdom

${ }^{51}$ STFC Rutherford Appleton Laboratory, Didcot, United Kingdom

${ }^{52}$ School of Physics and Astronomy, University of Edinburgh, Edinburgh, United Kingdom

${ }^{53}$ School of Physics and Astronomy, University of Glasgow, Glasgow, United Kingdom

${ }^{54}$ Oliver Lodge Laboratory, University of Liverpool, Liverpool, United Kingdom

${ }^{55}$ Imperial College London, London, United Kingdom

${ }^{56}$ School of Physics and Astronomy, University of Manchester, Manchester, United Kingdom

${ }^{57}$ Department of Physics, University of Oxford, Oxford, United Kingdom

${ }^{58}$ Massachusetts Institute of Technology, Cambridge, Massachusetts, USA

${ }^{59}$ University of Cincinnati, Cincinnati, Ohio, USA

${ }^{60}$ University of Maryland, College Park, Maryland, USA

${ }^{61}$ Syracuse University, Syracuse, New York, USA

${ }^{62}$ Pontifícia Universidade Católica do Rio de Janeiro (PUC-Rio), Rio de Janeiro, Brazil

(associated to Universidade Federal do Rio de Janeiro (UFRJ), Rio de Janeiro, Brazil)

${ }^{63}$ University of Chinese Academy of Sciences, Beijing, China

(associated to Center for High Energy Physics, Tsinghua University, Beijing, China) 


\author{
${ }^{64}$ School of Physics and Technology, Wuhan University, Wuhan, China \\ (associated to Center for High Energy Physics, Tsinghua University, Beijing, China) \\ ${ }^{65}$ Institute of Particle Physics, Central China Normal University, Wuhan, Hubei, China \\ (associated to Center for High Energy Physics, Tsinghua University, Beijing, China) \\ ${ }^{66}$ Departamento de Fisica, Universidad Nacional de Colombia, Bogota, Colombia \\ (associated to LPNHE, Université Pierre et Marie Curie, Université Paris Diderot, \\ CNRS/IN2P3, Paris, France) \\ ${ }^{67}$ Institut für Physik, Universität Rostock, Rostock, Germany \\ (associated to Physikalisches Institut, Ruprecht-Karls-Universität Heidelberg, Heidelberg, Germany) \\ ${ }^{68}$ National Research Centre Kurchatov Institute, Moscow, Russia \\ (associated to Institute of Theoretical and Experimental Physics (ITEP), Moscow, Russia) \\ ${ }^{69}$ Instituto de Fisica Corpuscular, Centro Mixto Universidad de Valencia-CSIC, Valencia, Spain \\ (associated to ICCUB, Universitat de Barcelona, Barcelona, Spain) \\ ${ }^{70}$ Van Swinderen Institute, University of Groningen, Groningen, Netherlands \\ (associated to Nikhef National Institute for Subatomic Physics, Amsterdam, Netherlands)
}

\footnotetext{
${ }^{\dagger}$ Deceased

${ }^{\mathrm{b}}$ Laboratoire Leprince-Ringuet, Palaiseau, France.

${ }^{\mathrm{d}}$ Università di Bari, Bari, Italy.

${ }^{\mathrm{e} U n i v e r s i t a ̀ ~ d i ~ B o l o g n a, ~ B o l o g n a, ~ I t a l y . ~}$

${ }^{\mathrm{f}}$ Università di Cagliari, Cagliari, Italy.

${ }^{\mathrm{g}}$ Università di Ferrara, Ferrara, Italy.

${ }^{\mathrm{h}}$ Università di Genova, Genova, Italy.

${ }^{\mathrm{i}}$ Università di Milano Bicocca, Milano, Italy.

${ }^{j}$ Università di Roma Tor Vergata, Roma, Italy.

${ }^{\mathrm{k}}$ Università di Roma La Sapienza, Roma, Italy. Poland.

${ }^{\mathrm{m}}$ LIFAELS, La Salle, Universitat Ramon Llull, Barcelona, Spain.

${ }^{\mathrm{n}}$ Hanoi University of Science, Hanoi, Viet Nam.

${ }^{\circ}$ Università di Padova, Padova, Italy.

${ }^{\mathrm{p}}$ Università di Pisa, Pisa, Italy.

${ }^{\mathrm{q}}$ Università degli Studi di Milano, Milano, Italy.

${ }^{\mathrm{r}}$ Università di Urbino, Urbino, Italy.

${ }^{\mathrm{s}}$ Università della Basilicata, Potenza, Italy.

${ }^{\mathrm{t}}$ Scuola Normale Superiore, Pisa, Italy.

${ }^{\text {u}}$ Università di Modena e Reggio Emilia, Modena, Italy.

${ }^{\vee}$ Iligan Institute of Technology (IIT), Iligan, Philippines.

${ }^{\mathrm{w}}$ Novosibirsk State University, Novosibirsk, Russia.
}

${ }^{a}$ Universidade Federal do Triângulo Mineiro (UFTM), Uberaba-MG, Brazil.

${ }^{c}$ P. N. Lebedev Physical Institute, Russian Academy of Science (LPI RAS), Moscow, Russia.

${ }^{1}$ AGH-University of Science and Technology, Faculty of Computer Science, Electronics and Telecommunications, Kraków, 